\title{
Active Detectors for Plasma Soft X-Ray Detection at PALS
}

\author{
C. Granja, V. Linhart, M. Platkevič, J. Jakůbek, \\ E. Krouský, S. Pospíšil, O. Renner, T. Slavíček
}

\begin{abstract}
This paper summarizes the work carried out for an experimental study of low-energy nuclear excitation by laser-produced plasma at the PALS Prague laser facility. We describe the adaptation and shielding of single-quantum active radiation detectors developed at IEAP CTU Prague to facilitate their operation inside the laser interaction chamber in the vicinity of the plasma target. The goal of this effort is direct real-time single-quantum detection of plasma soft X-ray radiation with energy above a few $\mathrm{keV}$ and subsequent identification of the decay of the excited nuclear states via low-energy gamma rays in a highly radiative environment with strong electromagnetic interference.
\end{abstract}

Keywords: laser induced nuclear excitation, laser-produced plasma, electromagnetic interference, photonuclear excitation.

\section{Introduction}

The possibility to excite low-energy nuclear states by laser-induced plasma has been initiated and experimentally investigated [1] on the Prague Asterix Laser System (PALS - see http://www.pals.cas.cz). This medium-energy high-power system yields interaction intensities at the level of $10^{16}-10^{17} \mathrm{Wcm}^{-2}$, which produce subrelativistic plasmas with an electron temperature of the order of 1-10 keV. Such laser-produced plasma (LPP) can excite low-lying nuclear states. Based on a systematic survey of suitable candidate nuclei [2], experiments have been undertaken to investigate the possible LPP excitation and $6 \mu$ s decay of $6.2 \mathrm{keV}$ level in ${ }^{181} \mathrm{Ta}$.

In framework of this project, particular effort has been devoted to the use of active detectors (i.e. realtime digital single-quantum counting devices) inside the interaction chamber for direct detection of plasma radiation and/or subsequent nuclear radiation. Two types of detector systems have been adapted for this purpose: hybrid semiconductor pixel detectors, and fast scintillating detectors.

In addition to the rather complicated target (Ta, W) and experimental setup (direct target observation or indirect geometry using either a secondary target as an ion collector or a toroidally bent crystal X-ray spectrometer to monochromatize the radiation emitted from the plasma), a major challenge was to shield the detector systems against scattered Xray radiation, high-energy particles, and in particular against the strong electromagnetic interference (EMI) induced by the laser pulse. We report the development of special instruments complying with crude requirements for detecting the delayed X-ray emission close to the laser-irradiated targets, and we outline future work.

\section{Laser-produced plasma nuclear interaction}

\subsection{Plasma radiation at PALS}

The PALS medium-size high-power laser system [3] (fundamental laser wavelength $1.315 \mu \mathrm{m}$, pulse duration $250 \mathrm{ps}$ ) provides strong light pulses with energies at the level of several hundreds of Joules and at subrelativistic intensities above $10^{16} \mathrm{Wcm}^{-2}$. The characteristics of the plasma emission accompanying the laser-matter interaction depend on the laser pulse parameters and the particular target material that is used.

By irradiating a ${ }^{181} \mathrm{Ta}$ target with a laser intensity of $7 \cdot 10^{16} \mathrm{Wcm}^{-2}$, the plasma electron temperature near the target surface exceeds $1 \mathrm{keV}$ at electron density close to $10^{21} \mathrm{~cm}^{-3}$, the number of ions in the whole plasma volume (about $3 \cdot 10^{-6} \mathrm{~cm}^{-3}$ ) is estimated at $4 \cdot 10^{13}$, and their average charge can reach $40[2,4,5]$.

\subsection{Plasma-induced nuclear excitation}

Plasma-nuclei interactions in hot, dense LPP can result in nuclear-photonic and nuclear-electronic excitations $[6,7]$ such as direct photoexcitation and nuclear excitation by electronic transition (NEET). With the plasma characteristics achievable at PALS, we consider these two processes as dominant mechanisms for low-energy nuclear excitation $[2,4]$.

\subsubsection{Nuclear de-excitation}

The decay of low-energy excited levels in nuclei ${ }^{181} \mathrm{Ta}$ $(6.238 \mathrm{keV}, 6.05 \mu \mathrm{s})$ proceeds via emission of $\gamma$ rays or via internal electron conversion (IC) [2]. With decreasing transition energy $(E \gamma<100 \mathrm{keV})$, the latter mechanism becomes increasingly dominant. However, atomic shell ionization can significantly affect the decay rate and the decay branching ratio [2]. 


\section{Active detectors for direct detection of plasma radiation}

Two types of active detector systems are being implemented and adapted for direct detection of plasma radiation (see http://www.utef.cvut.cz): (i) hybrid semiconductor pixel detectors of the Medipix type (developed in the framework of Medipix Collaboration [8]), and (ii) fast scintillating detectors (of which $\mathrm{BaF}_{2}$ was selected as the most suitable type).

\subsection{Medipix2/USB camera}

State-of-the-art hybrid pixel semiconductor detectors of the Medipix family [8] are characterized by spatial, energetic and temporal resolution which make them attractive for nuclear and particle spectroscopy [9]. In addition to the position-sensitive and single quantum detection capability of Medipix2 [10], the new TimePix device [11] also provides the possibility to determine the detection time and/or the energy deposition in each individual pixel.

Real-time operation of detectors of this type, and also data readout and on-line visualization are realized via the MUROS interface [12], or with the integrated USB-based readout interface [13], which links by a standard USB port into any PC and provides the necessary power supply (see http://www.utef.cvut.cz/medipix). Operation and control of the system, as well as data acquisition, are driven by a Windows-compatible Pixelman software package [14]. Data stream acquisition and storage proceed on-line at an overall rate of about 5 frames per second.

The assembled TimePix/USB device [15] has dimensions $142 \times 50 \times 20 \mathrm{~mm}^{3}$ (shown in Figure 1), and serves as a versatile portable real-time hand-held radi-

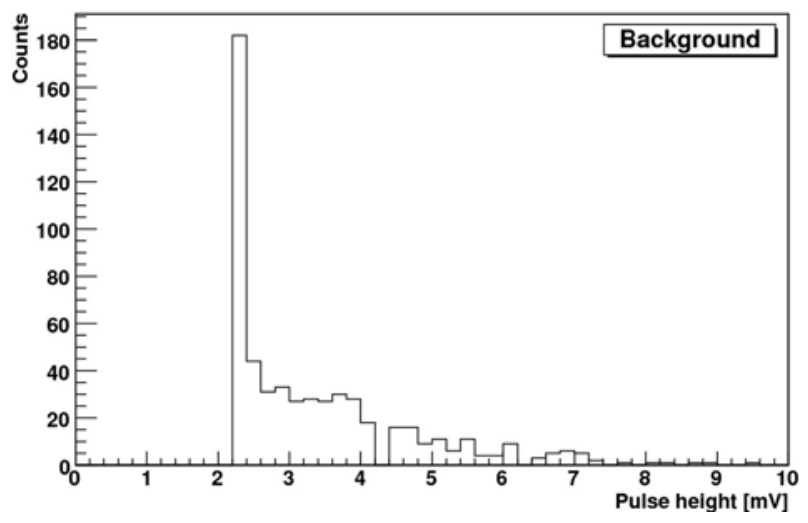

ation camera of multi-particles (X-rays, $e, p, \alpha, t$, ions, $n)$ with position-, time- and energy-sensitive capability for single-quantum and on-line particle and nuclear spectroscopy [9].

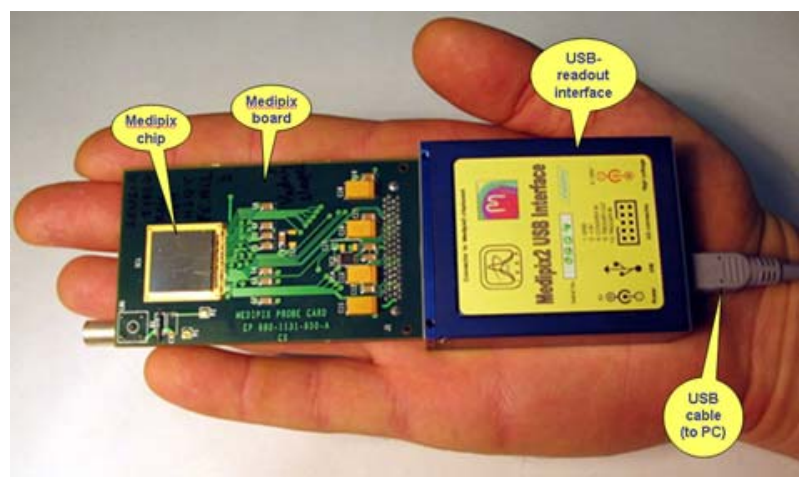

Fig. 1: Medipix2/USB-interface radiation camera. The Medipix board (left) is attached to the USB-based readout interface (right)

\section{2 $\quad \mathrm{BaF}_{2}$ scintillating detector for soft $\mathrm{X}$-ray detection}

We investigated the spectrometric response of a $\mathrm{BaF}_{2}$ scintillating detector (Korth Kristalle $\mathrm{GmbH}$ ) of dimensions $5 \times 5 \times 5 \mathrm{~mm}^{3}$, decay time $630 \mathrm{~ns}$ (slow component) resp. $0.6-0.8 \mathrm{~ns}$ (fast component), light yield 10 photons $/ \mathrm{keV}$ (slow) resp. 1.8 photons $/ \mathrm{keV}$ (fast) and photoelectron $\mathrm{NaI}(\mathrm{Tl})$ light yield of $16 \%$ (slow) resp. $3 \%$ (fast). A fast photomultiplier (Hamamatsu No. H5783-04) was used. The energy threshold of this system was determined at $1 \mathrm{keV}$ (noise level) corresponding to an amplitude of $2 \mathrm{mV}$. Tests done with $5.9 \mathrm{keV}$ X-rays from ${ }^{55} \mathrm{Fe}$ yield a maximum at $12 \mathrm{mV}$ with a mean measured signal of about $4 \mathrm{mV}$. The amplitude spectra collected with this radioactive source are shown in Figure 2.

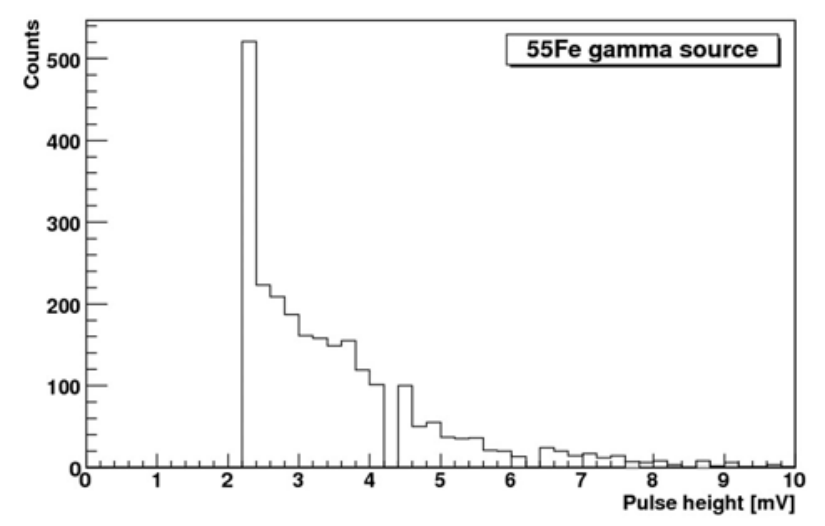

Fig. 2: Amplitude spectra of background (left) and ${ }^{55} \mathrm{Fe}$ source (right) collected with the $\mathrm{BaF}_{2}$ detector 


\section{Detector shield for very high radiation and EMI}

Experimental conditions for generating LPP are characterized by (i) a high vacuum, (ii) a very high radiation background, and (iii) extremely high electromagnetic interference (EMI) in the interaction chambers. The noise accompanying the interaction of the high power laser beams with the matter results in significant challenges for the operation and effective shielding of active detectors inside such hostile environments.

\subsection{Semiconductor Pixel Detector}

First of all, the detector chip board and the USBinterface were implemented and tested for operation in a high vacuum. Moreover, background radiation and specially EMI noise were expected to affect the operation of the detector chip, which consists of a bump bonded detector sensor and a microelectronics chip with highly integrated components (preamplifier, ADC convertor, digital counter) for each of the 65000 pixels which act as individual detectors.

\subsubsection{Medipix2/USB-interface inside the chamber}

In the first stage, we attempted to keep both the detector board and the readout interface as one unit (see Figure 1) operating inside the interaction chamber [16]. In view of the expected high radiation and high electromagnetic noise, the detector system was doubly shielded. The first shield consisted of an aluminum casing with a cylindrical tube in front of the active sensor chip of the detector connected to ground. The second shield, isolated from the first shield, consisted of a lead-coated plate grounded to the chamber. The communication and power supply cables were also double shielded. In spite of the shielding, the laser pulse still affected the power supply and reset the CPU of the interface. This problem was solved by applying a DC-DC converter $(20 \mathrm{~V}$ to $5 \mathrm{~V})$ and a filtering capacitor close to the device. This scheme was functional at energies of the laser shots up to about 10 J. At more powerful flashes, the USB communication failed. We fixed this problem by disconnecting the data wires during the measurement by relay. The corresponding modification of the software was also implemented. The measurement procedure required preprogramming the device to wait for a trigger, disconnecting the USB data wires (by relay), and after the laser shot, reconnecting the USB data wires, and reading out the data. However, this solution was not reliable, as it was only occasionally functional with laser energies up to about $50 \mathrm{~J}$.

\subsubsection{Medipix board detached from the USB-interface by a devoted module}

Since strong effects of EMI persisted, and in an effort to minimize the number of components in the very high field noise inside the interaction chamber, the USB-readout interface (of dimensions $64 \times 50 \times$ $20 \mathrm{~mm}^{3}$ ) was physically distanced from the Medipix detector chip board. This was attained by constructing a radiation resistant single-purpose communication LVDS module (see Figure 3) with separate data communication (three Ethernet cables) and power lines between the detector board (placed inside the interaction chamber) and the USB-readout interface (outside the chamber) [17]. A power supply to the module and to the detector board was provided for each device separately.

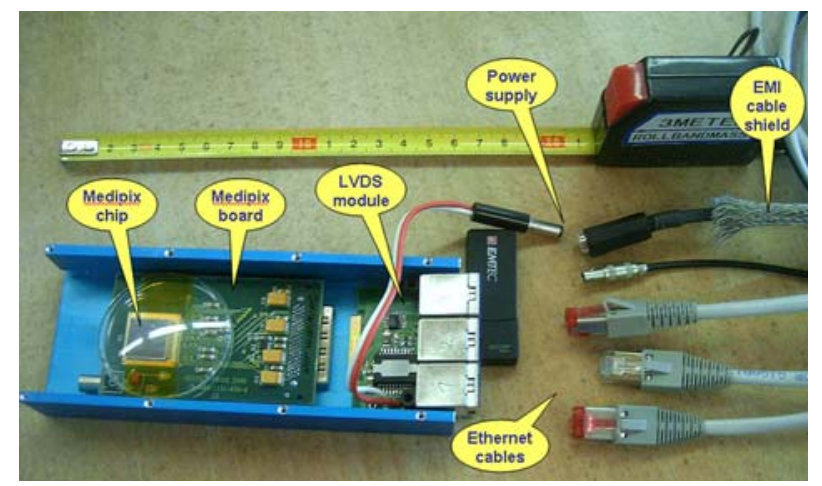

Fig. 3: Medipix2 detector and LVDS Module with communication ethernet and power cables. The EMI shield for one cable is shown

While the Medipix detector board freely operates in a vacuum, namely the heat in the LVDS module has to be dissipated. For this purpose, we glued the bottom side of the module (and also that of the detector board - to ensure its long-term stability) to an aluminum panel using a thermo-conductive polymer (silicone elastomer Sylgard 160).

In a subsequent step, in view of the strong electromagnetic interference (EMI) with very high and short gradients, a special EMI shielding assembly was designed and constructed for all components to operate inside the interaction chamber [17]. Communication and power cables were EMI protected by a flexible shielding insulating tube through which a cable or a bundle of cables is conveyed. The material used is $\mu$-copper for both electrical and magnetic screening (Holland Shielding). The inner EMI shielding was grounded to earth. The outer EMI shield was connected to the interaction chamber wall. The detector board was also EMI shielded by a gradual onion-like assembly of several (up to three) independent screening arrangements of reinforced Amucor and mu-copper foils (hollandshielding.com). These successive layers 
were insulated from each other by additional insulating material. The ground was taken out independently by the single connection to avoid induced loops. The interface and contacts between the cable shielding tubes and the detector board shield casing were secured by EMI insulating metal conductive tapes. To ensure reliability of operation, the cabling and the USB-based interface outside the interaction chamber were also EMI shielded.

\subsection{Scintillating Detector}

In view of both the ionizing radiation background and the non-ionizing EMI noise, a massive and complex shield was required. EMI induces a marked but gradually varying disturbance of the detector/preamplifier signal. This occurs even before the arrival of the laser shot (see Figure 9 and Figure 10), as a consequence of the laser charging and laser resonance effects.

The design of the system shield against ionizing radiation was conceived [18] as three segments/layers: outer, intermediate and inner (see Figure 4). The outer shield consisted of $2 \mathrm{~cm}$-thick polyethylene with the aim of stopping or slowing down electrons emitted by the target. The intermediate layer consisted of dural foils with total thickness of $5 \mathrm{~mm}$ placed namely at the interfaces and loose edges of the surrounding shielding blocks. Their task was to stop the charged particles which eventually arise and cross the interblock apertures. The inner layer contained three lead sheet plates with a full thickness of about $15 \mathrm{~mm}$. They shielded the detector against photons originating directly from the plasma and/or from the interaction of the ion fluxes with the chamber and shielding blocks. This inner layer should already avoid any direct charged particle impact, with the exception of relativistic electrons. Further thick $\mathrm{Al}$ and $\mathrm{Pb}$ plates as well as thin $\mathrm{Al} / \mathrm{C}$ paper foils were additionally implemented. The detector window was protected by a light-tight $125 \mu \mathrm{m}$ thick Be foil. Mountable $\mathrm{Pb} / \mathrm{PE}$ collimators with bore opening $3 \mathrm{~mm}$ and $8 \mathrm{~mm}$ were also used (see inset in Figure 5).

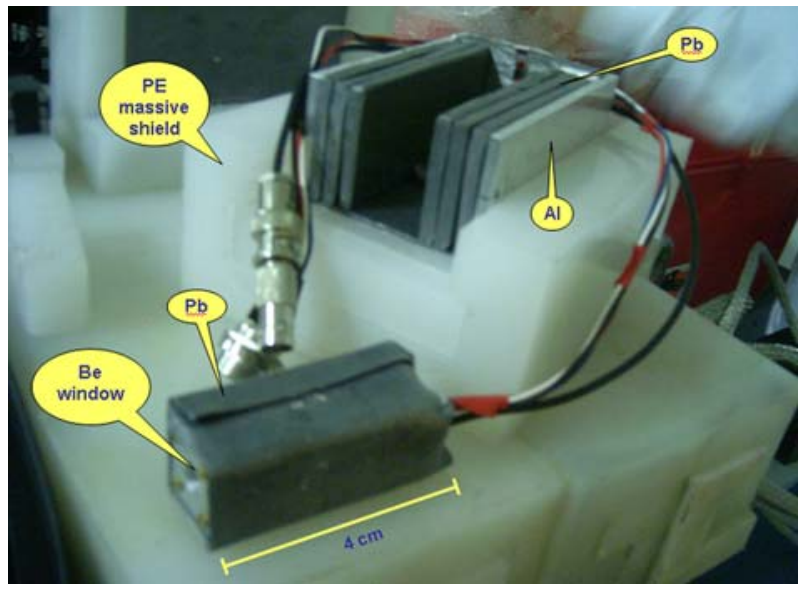

Fig. 4: $\mathrm{BaF}_{2}$ detector with lead $(\mathrm{Pb})$, Aluminum $(\mathrm{Al})$ and Polyethylene (PE) shield

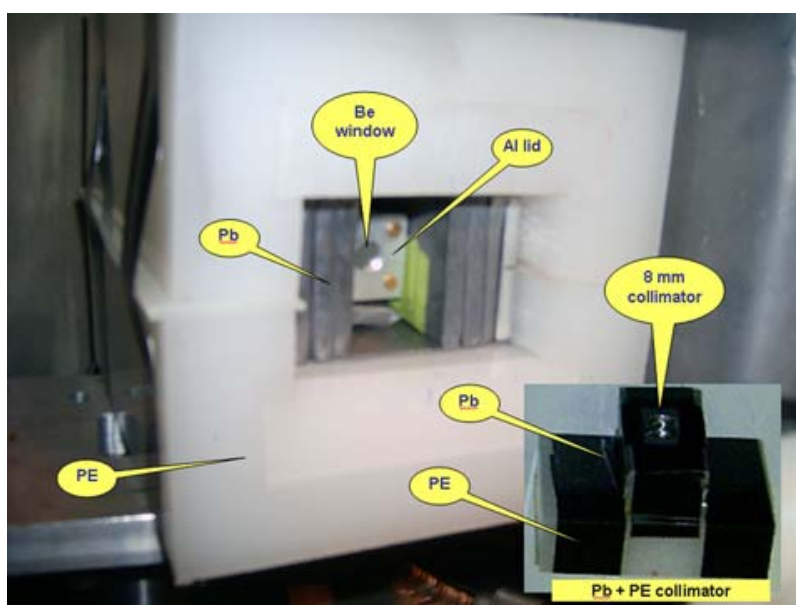

Fig. 5: Shield assembly for $\mathrm{BaF}_{2}$ detector with lead (Pb), Aluminum ( $\mathrm{Al})$ and Polyethylene (PE) segments. The $\mathrm{Pb} / \mathrm{PE}$ shield with $8 \mathrm{~mm}$ collimator is shown in the inset

\section{Measurements at PALS}

The experiments were carried out by irradiating massive Ta and $\mathrm{W}$ targets with the PALS frequencytripled single laser beam (50-250 J, $0.44 \mu \mathrm{m}, 250 \mathrm{ps,}$ $\left.0.4-2 \cdot 10^{16} \mathrm{Wcm}^{-2}\right)$; the laser characteristics were checked by a routine diagnostic complex [3]. For the search of $6.2 \mathrm{keV}$ excitation in ${ }^{181} \mathrm{Ta}$, the highest fluence of plasma X-ray radiation is estimated [4] for laser energies around $150 \mathrm{~J}$.

\subsection{Medipix2}

For the operation of the Medipix detector in this hostile environment, a number of different, and increasingly extended, shielding setups were assembled and tested. In addition to the special hardware implementation (Figure 3), we tested a number of EMI shielding arrays which were gradually assembled in consecutive layers/circuits. Operation of the detector inside the interaction chamber was ultimately achieved with a complex three-independent circuit EMI shield. The fully assembled system in the interaction chamber is shown in Figure 6.

Measurements were carried out using two different targets ( $\mathrm{Ta}$ and $\mathrm{W}$ ) with the shielding assembly described above. The detector managed to remain operational at the desired laser energies (170 J). However, in view of the relatively short decay time $(6 \mu \mathrm{s})$ of the excited level $(6.2 \mathrm{keV})$ in ${ }^{181} \mathrm{Ta}$, and the duration of the laser plasma flash pulse (250 ps), the additional use of a trigger should guarantee separation of the expected spectrometric signals from the large primary laser pulse and subsequent intense plasma radiation. 


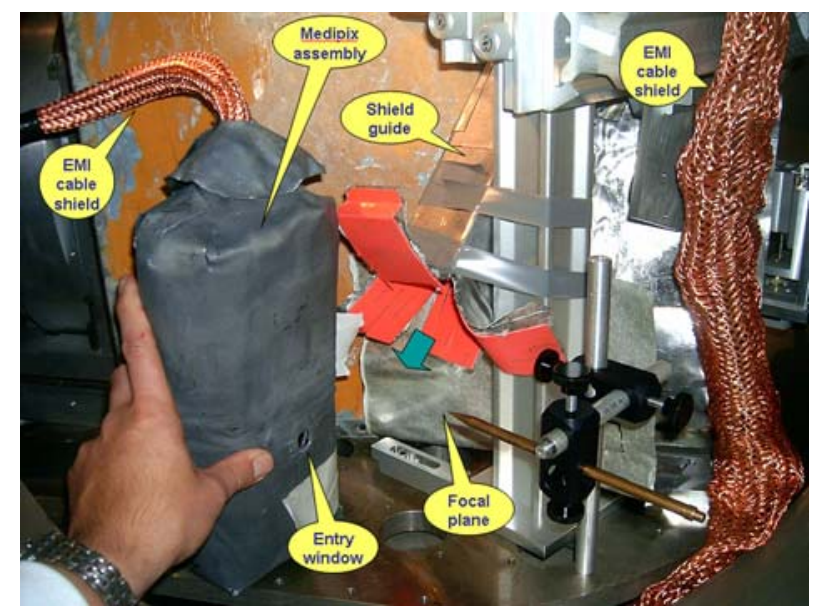

Fig. 6: Medipix detector and shielding assembly setup in the PALS interaction chamber. The diffracted X-rays (green arrow) coming from the bent crystal are detected by the detector placed at the focal plane

\section{2 $\quad \mathrm{BaF}_{2}$ detector}

With this detector, measurements were carried out in various experimental and shielding setups. In terms of target and detector position we tested a direct view setup, where the detector window is directed at the primary target, and an indirect view setup, where the detector faces either the curved crystal (Figure 6) or a secondary target functioning as an ion collector (Figure 7). Measurements were performed with different massive shielding arrays, which are illustrated in Figure 8. The shield assembly for this detector was composed of blocks/layers made of polyethylene (PE), lead $(\mathrm{Pb})$, aluminum $(\mathrm{Al})$ of different segment sizes and thicknesses (see Figure 8). A thin beryllium (Be) foil window covered the detector window.

\subsubsection{Direct view on target}

While the EMI noise was significantly suppressed, even this fully closed and massive shield yielded background signals, as shown in Figure 9, for a Ta Target. Results of similar response tests obtained with varying laser energy, from zero up to $157 \mathrm{~J}$, are shown in Figure 10. In this geometry, even this strong shield is not sufficient, and the penetrating radiation remains non-negligible (i.e. relativistic electrons and/or high energy gamma rays).

\subsubsection{Indirect view on secondary target-collector}

In this geometry, a massively protected detector with a sealed aperture was used. The space between the target and the detector was complementarily shielded by massive lead plates. However, opening the aperture even with varying collimator size and composition resulted in a strongly disturbed response. The response measurements are illustrated in Figure 11.

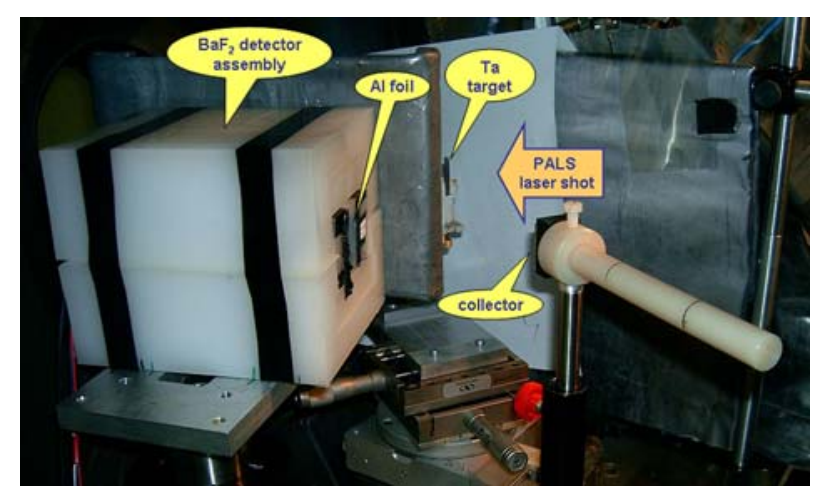

Fig. 7: Indirect experimental setup in the PALS interaction chamber: The $\mathrm{BaF}_{2}$ detector assembly, facing the secondary target - the collector, is shielded from the primary Ta target

\subsubsection{Indirect view behind the monochromator crystal}

With the toroidally bent crystal spectrometer, the detector response yielded similar results as . Collimated apertures simply did not fully shield the detector. At least a thin plate of $\mathrm{Pb}$ and $\mathrm{Al}$ were required, even in this geometry.

\section{Conclusions}

For the laser parameters and Ta target used here, plasma-penetrating radiation remains the principal noise factor for scintillating detectors, while EMI noise was the most important limiting factor for semiconductor pixel detectors. The possibility of successfully applying both types of detectors has been demonstrated. In future work, the timing of Medipix data collection at the desired time interval (a few $\mu$ s after the laser flash) should be driven by the laser trigger, which is usually generated $4-5 \mu$ s before the laser shot. The $\mathrm{BaF}_{2}$ detector is most suitable for following the time evolution of the plasma radiation as a whole. This may proceed, e.g., via recording the complete assembly of the emitted quanta in consecutive time intervals (e.g. $5 \mathrm{~ns}$ ). Until now, the $\mathrm{BaF}_{2}$ detector could be used only for observing de-exciting nuclear radiation from indirect targets. Moreover, the detector entrance window had to be at least partially shielded by thin $\mathrm{Pb} / \mathrm{Al}$ foils, which significantly reduced the overall detection efficiency of the desired radiation. Work on further improving and stabilizing the operation of these detectors in such a radiation and EMI noise environment is in progress. 

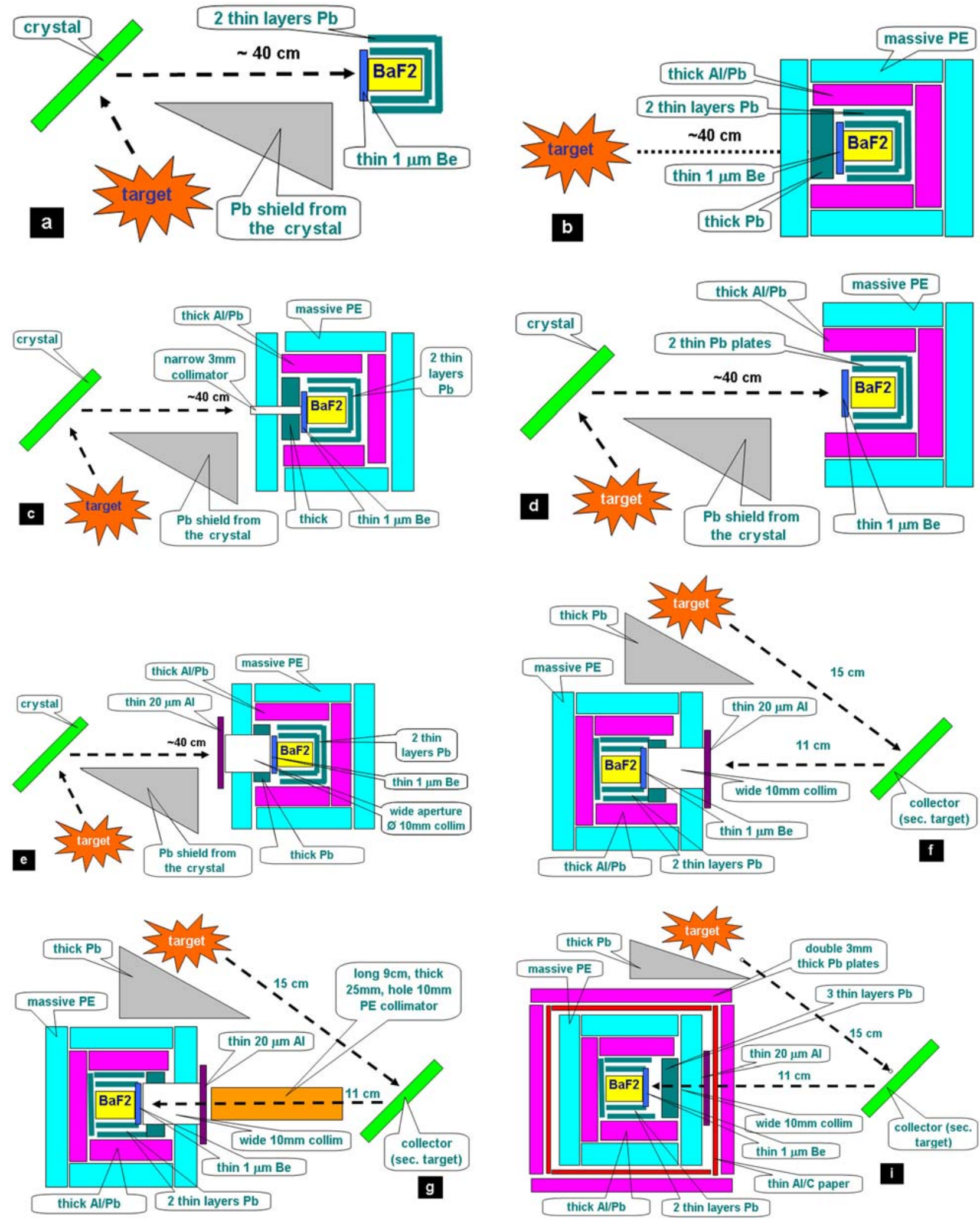

Fig. 8: Schematic illustration of the target and shield setups investigated for the $\mathrm{BaF}_{2}$ detector. The geometric setup is determined by the position of the primary target, the (eventual) crystal/collector as a secondary target, and the detector. The shield assembly was composed of materials with polyethylene ( $\mathrm{PE})$, lead $(\mathrm{Pb})$, Aluminum ( $\mathrm{Al})$, a thin Beryllium (Be) foil window in various segment sizes and thicknesses 

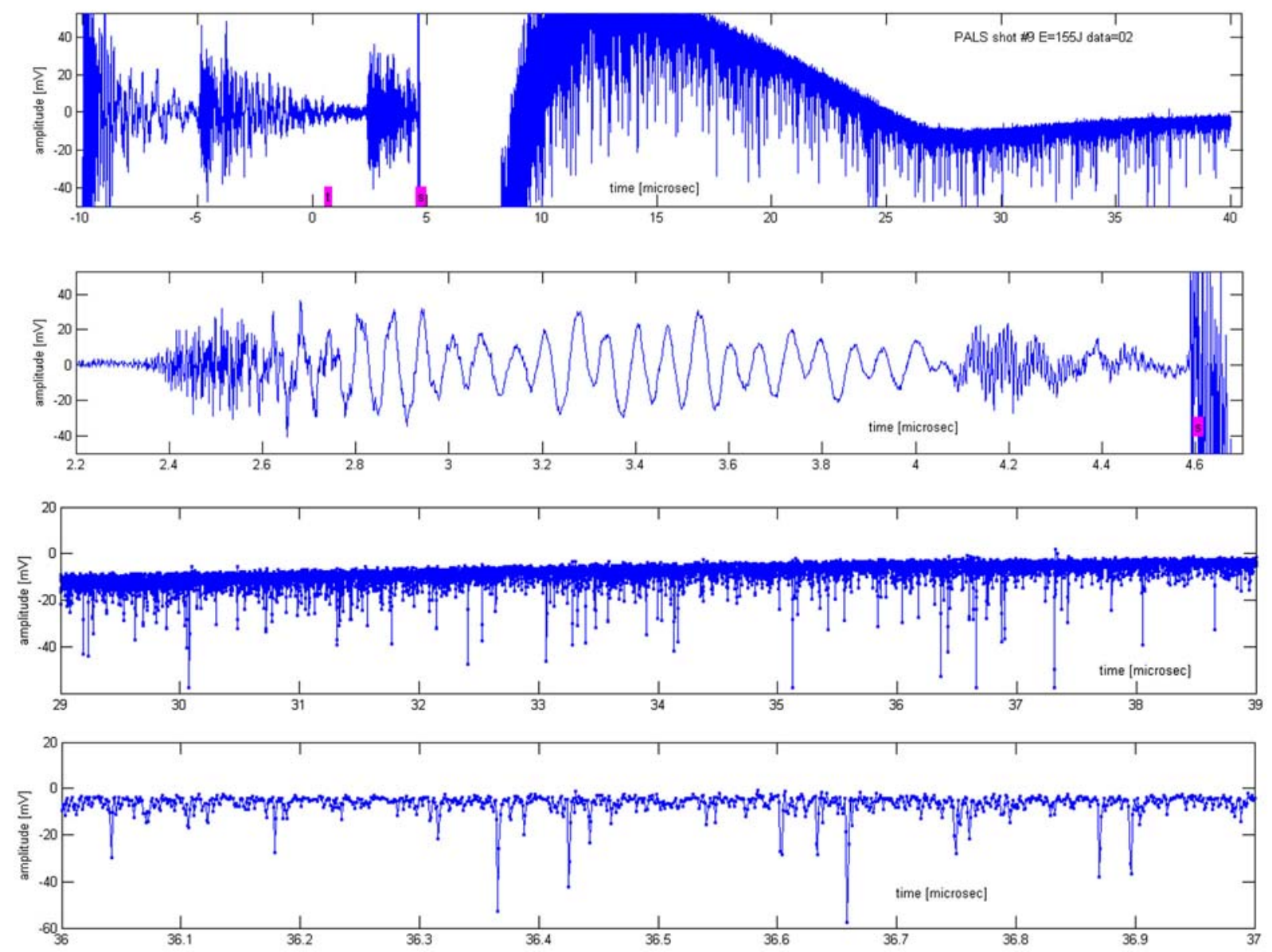

Fig. 9: Time spectrum of a laser shot (\#09) with energy $155 \mathrm{~J}$ on a Ta target in setup "a" (see Figure 8). The full spectrum is shown (top). For illustration, parts of the spectrum are shown in detail: prior and at the laser shot (second from top), at a later time - the region between $29 \mu \mathrm{s}$ and $39 \mu \mathrm{s}$ (third from top) as well as a region between $36 \mu \mathrm{s}$ and $37 \mu \mathrm{s}$ (bottom). The laser shot time and the PALS laser trigger signal level (generally at about $4 \mu$ s before the laser shot) are indicated by letters "s" and "t", respectively

\section{Acknowledgement}

This work was funded by Research Grant No. 202/06/0697 of the Czech Science Foundation, and was carried out in framework of Research Program MSM 6840770029 of the Czech Ministry of Education, Youth and Sports.

\section{References}

[1] Granja, C., Jakůbek, J., Linhart, V., Pospíšil, S., Slavíček, T., Uher, J., Vykydal, Z., Kuba, J., Sinor, M., Drska, L., Renner, O., Juha, L., Krása, J., Krouský, E., Pfeifer, M., Ullschmied, J.: Search for low-energy nuclear transitions in laserproduced plasma, Czech. J. Phys., 56, 2006, p. $478-484$.

[2] Granja, C., Haiduk, A., Kuba, J., Renner, O.: Survey of nuclei for low-energy nuclear excitation in laser-produced plasma, Nuclear Physics A, 784, 2007, p. 1-12.
[3] Jungwirth, K., et al.: The Prague Asterix Laser System PALS, Physics of Plasmas, 8, 2001, p. 2495 .

[4] Renner, O., Juha, L., Krása, J., Krouský, E., Pfeifer, M., Velyhan, A., Granja, C., Jakůbek, J., Linhart, V., Slavíček, T., Vykydal, Z., Pospíšil, S., Kravařík, J., Ullschmied, J.: Low-energy nuclear transitions in subrelativistic laser-generated plasmas, Laser and Particle Beams, 26, 2008, p. 249-257.

[5] Renner, O., Granja, C., Linhart, V., Pospíšil, S., Juha, L., Krása, J., Krouský, E.: Search for low-energy nuclear transitions in laser-produced plasma, Proc. EPS Plasma Physics Conference, Vol. 32, Plasma Physics and Controlled Fusion Series, Bristol, Institute of Physics Publishing, 2008, p. $126-131$. 

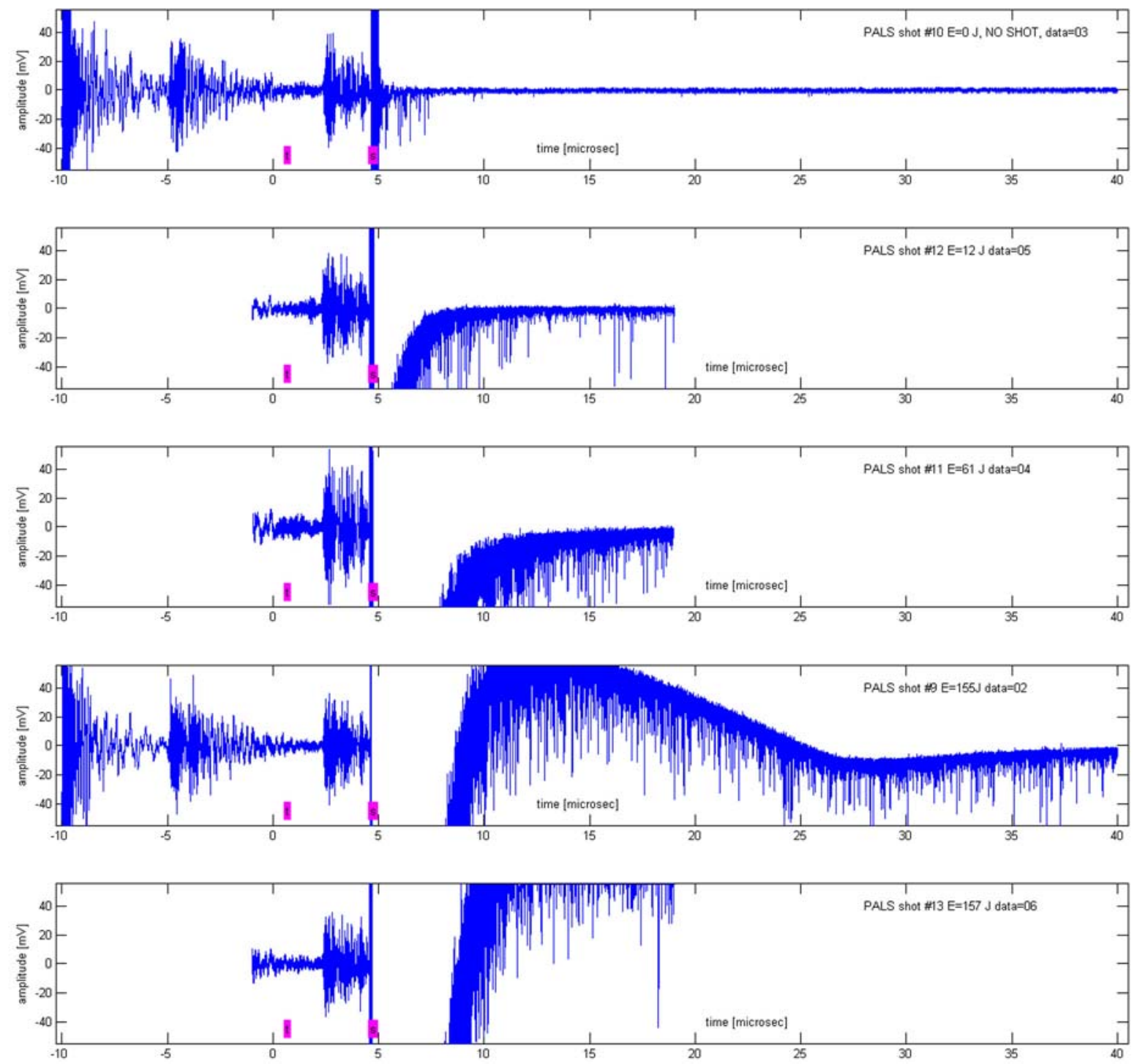

Fig. 10: Time spectra collected on the Ta target in setup "a" (see Figure 8) for varying laser pulse energy: (from top to bottom) no shot, 12 J, $61 \mathrm{~J}, 155 \mathrm{~J}$ (same as Figure 9) and $157 \mathrm{~J}$. Trigger and shot level are indicated. Time channel/range settings varied for some measurements

[6] Harston, M. R., Chemin, J. F.: Mechanisms of nuclear excitation in plasmas, Phys. Rev. C, 59 (5), 1999, p. 2462-2473.

[7] Tkalya, E. V.: Mechanisms for the excitation of atomic nuclei in hot dense plasma, Laser Physics, 14, 2004, p. 360-377.

[8] See http://www.cern.ch/medipix and http://www.utef.cvut.cz/medipix

[9] Granja, C., Vykydal, Z., Jakůbek, J., Pospíšil, S.: Position-Sensitive Nuclear Spectroscopy with Pixel Detectors, Conf. Proc. Series, Vol. 947, New York, American Institute of Physics, 2007, p. $449-452$.

[10] Llopart, X., Campbell, M., Dinapoli, R., San Segundo, D., Pernigotti, E.: Medipix2 - a $64 \mathrm{k}$ pixel readout chip with $55 \mu \mathrm{m}$ square elements working in single photon counting mode, Proc. IEEE NSS/MIC, Trans. Nucl. Sci., 49, San Diego, IEEE, 2002, p. 2279.

[11] Llopart, X., Ballabriga, R., Campbell, M., Wong, W.: Timepix, a $65 \mathrm{k}$ programmable pixel readout chip for arrival time, energy and/or photon counting measurements, Nuclear Instruments and Methods in Physics Research A, 585, 2007, p. $106-108$.

[12] San Segundo Bello, D., Van Beuzekom, M., Jansweijer, P., Verkooijen, H,. Visschers, J.: An interface board for the control and data acquisition of the Medipix2 chip, Nuclear Instruments and Methods in Physics Research A, 509, 2003, p. $164-170$. 

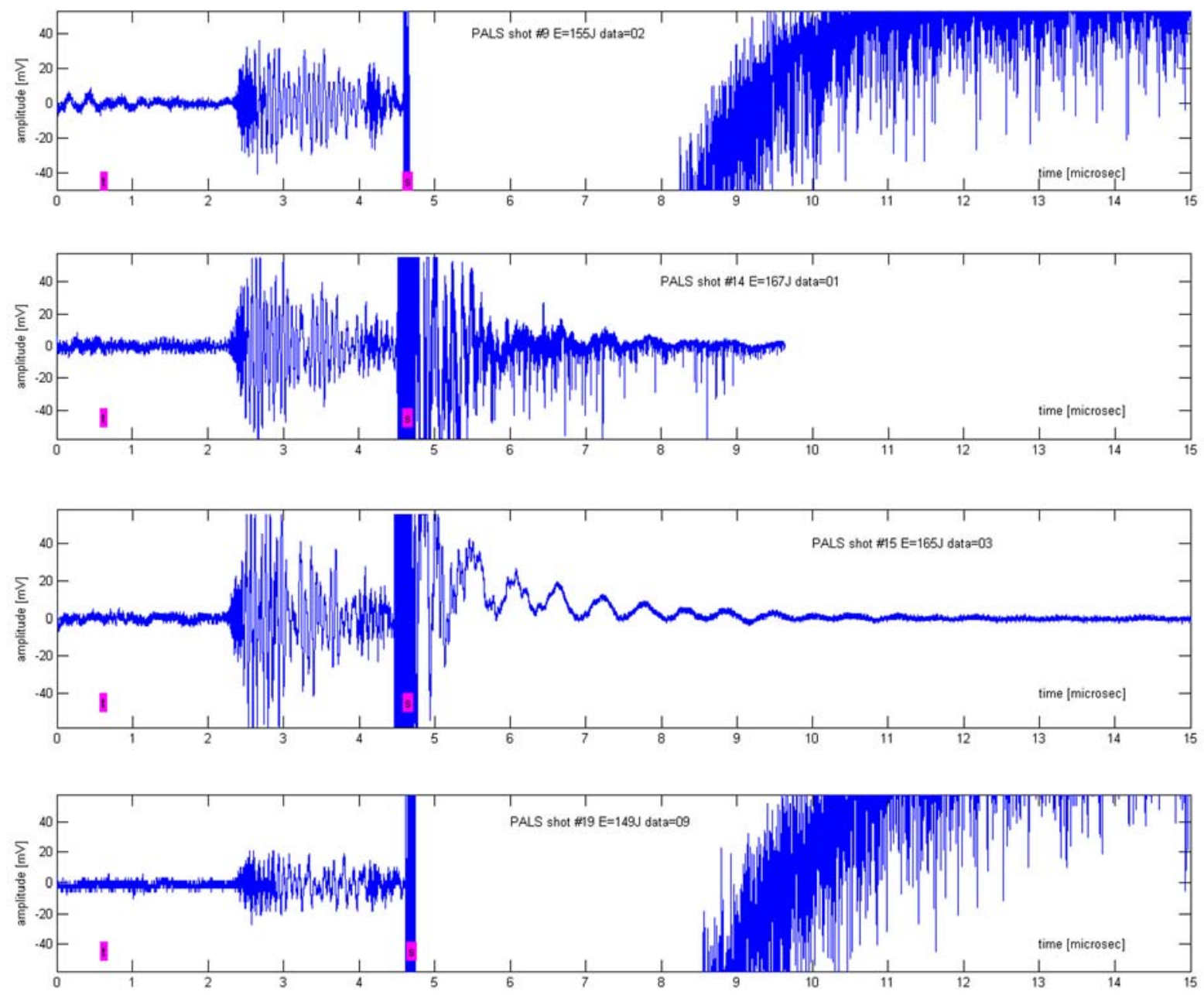

Fig. 11: Time spectra collected on a Ta target (similar as Figure 9) at time range $0 \mu$ s to $15 \mu$ s for four different detector shield setup settings (from top to bottom: "a", "b", "c" and "e" - see Figure 8). The laser shot energy (in joules) is indicated and was very similar for these graphs $\left(155 \mathrm{j}, 167 \mathrm{j}, 165 \mathrm{j}\right.$ and $149 \mathrm{j}$; all in the $3^{\text {rd }}$ harmonic). The PALS laser trigger and shot signal levels (at about $0.7 \mu \mathrm{s}$ and $4 \mu \mathrm{s}$, respectively) are labeled by the red boxes. The Ta target is the same. Time channel/range settings varied for some measurements (e.g., "b")

[13] Vykydal, Z., Jakůbek, J., Pospíšil, S.: USB interface for Medipix2 enabling energy and position detection of heavy-charged particles, Nuclear Instruments and Methods in Physics Research A, 563, 2006, p. 112-115.

[14] Holý, T., Jakůbek, J., Pospíšil, S., Uher, J., Vavrik, D., Vykydal, Z.: Data acquisition and processing software package for Medipix2, $\mathrm{Nu}$ clear Instruments and Methods in Physics Research A, 563, 2006, p. 254-258.

[15] Vykydal, Z., Jakůbek, J., Holý, T., Pospíšil, S.: A portable pixel detector operating as an active nuclear emulsion and its application for X-ray and neutron tomography, Proc. $9^{\text {th }}$ Conf. on astroparticle, particle and space physics, detectors and medical applications, Como, World Scientific, 2006, p. 779-784.

[16] Platkevič, M., Granja, C., Jakůbek, J., Vykydal, Z.: Medipix in extremely hostile environment, Proceedings Workshop CTU Prague, Vol. 12, No. ELP018, 2008, p. 212-213.

[17] Platkevič, M., Granja, C, Jakůbek, J., Pospíšil, S.: Electromagnetic Interference Shielding for Medipix Detectors for Laser-Induced Plasma Radiation Detection, Proceedings Workshop CTU Prague, 2009, in print.

[18] Granja, C., Linhart, V., Pospíšil, S., Slavíček, T.: Scintillating BaF2 Detector for Low-Energy Nuclear Excitation at PALS, Proceedings Workshop CTU Prague, 2009, in print. 
Doc. Ing. Carlos Granja, Ph.D.*

Phone: +420 224359394

E-mail: carlos.granja@utef.cvut.cz http://www.utef.cvut.cz

[*Corresponding Author]

Institute of Experimental and Applied Physics

Czech Technical University in Prague

Horská 3a/11, 12800 Prague 2, Czech Republic

Ing. Vladimír Linhart, Ph.D.

Phone: +420224359180

E-mail: Vladimir.Linhart@utef.cvut.cz

Institute of Experimental and Applied Physics

Czech Technical University in Prague

Horská 3a/11, 12800 Prague 2, Czech Republic

[current address - University of Valencia, Spain]

Ing. Michal Platkevič

Phone: +420224359181

E-mail: michal.platkevic@utef.cvut.cz

Institute of Experimental and Applied Physics

Czech Technical University in Prague

Horská 3a/11, 12800 Prague 2, Czech Republic

Jan Jakůbek

Phone: +420224359181

E-mail: jan.jakubek@utef.cvut.cz

Institute of Experimental and Applied Physics

Czech Technical University in Prague

Horská 3a/11, 12800 Prague 2, Czech Republic prom. fyz. Eduard Krouský, CSc.

Phone: +420266052136

E-mail: krousky@fzu.cz

Institute of Physics, v.v.i.

Academy of Sciences of the Czech Republic

Na Slovance 2, 18221 Prague 8, Czech Republic

Stanislav Pospíšil

Phone: +420224359290

E-mail: stanislav.pospisil@utef.cvut.cz

Institute of Experimental and Applied Physics

Czech Technical University in Prague

Horská 3a/11, 12800 Prague 2, Czech Republic

Ing. Oldřich Renner, DrSc.

Phone: +420266 052136

E-mail: renner@fzu.cz

Institute of Physics, v.v.i.

Academy of Sciences of the Czech Republic

Na Slovance 2, 18221 Prague 8, Czech Republic

Tomáš Slavíček

Phone: +420224359180

E-mail: Tomas.Slavicek@utef.cvut.cz

Institute of Experimental and Applied Physics

Czech Technical University in Prague

Horská 3a/11, 12800 Prague 2, Czech Republic 